Annals of Pure and Applied Mathematics

Vol. 18, No. 1, 2018, 59-64

ISSN: 2279-087X (P), 2279-0888(online)

Published on 6 August 2018

Annals of

www.researchmathsci.org

DOI: http://dx.doi.org/10.22457/apam.v18n1a8

Pure and Applied

Mathematics

\title{
Perfect Domination for Bishops, Kings and Rooks Graphs On Square Chessboard
}

\author{
K.S.P. Sowndarya and Y. Lakshmi Naidu \\ Department of Mathematics and Computer Science \\ Sri Sathya Sai Institute of Higher Learning, Anantapur Campus \\ Anantapur - 515001, Andhra Pradesh, India \\ Email: kspsowndarya44@gmail.com, ylakshminaidu@sssihl.edu.in
}

Received 4 July 2018; accepted 2 August 2018

\begin{abstract}
The chessboard domination problems were said to be origin of the study of graphs. The $\mathrm{n}^{2}$ cells in the chessboard of order $n \times n$ are treated as vertices and two vertices are adjacent if one can cover the other vertex in one move directly. Various studies had been done on these chessboard problems in relation to different domination parameters such as domination, independence and irredundance on queens, bishops, kings and rooks graphs. In this paper we extend this study on perfect domination and determine the exact values of Perfect Domination number for Bishops graph $\boldsymbol{B}_{\boldsymbol{n}}$, Kings Graph $K_{n}$, and Rooks Graph $R_{n}$ on an $\mathrm{n} \times \mathrm{n}$ chessboard.
\end{abstract}

Keywords: Chessboard Domination, Bishops Graph, Kings Graph, Rooks Graph, Perfect Domination number.

\section{AMS Mathematics Subject Classification (2010): 05C69}

\section{Introduction}

The number of squares in the entire chessboard of order $n \times n$ can be considered as vertices, while the edges are formed between two vertices or squares if they are adjacent. In Bishops graph $B_{n}$ two squares are adjacent if one square can cover the other in the same diagonal, whereas, in Kings graph $K_{n}$ two squares are adjacent if distance between two squares is one i.e., $d(u, v)=1$ where $u$ and $v$ are two squares, or each King on an $n \times n$ chessboard is said to attack its own square and its neighboring squares, i.e., the nine or fewer squares within one move of the King. In Rooks graph $R_{n}$ two squares are adjacent if they are in same row or column. A set $D$ of squares is a dominating set of $\mathrm{B}_{\mathrm{n}} /$ $\mathrm{K}_{\mathrm{n}} / \mathrm{R}_{\mathrm{n}}$ if every square of $\mathrm{B}_{\mathrm{n}} / \mathrm{K}_{\mathrm{n}} / \mathrm{R}_{\mathrm{n}}$ is either in $D$ or adjacent to a square in $D$, and the minimum cardinality of the dominating set of these graphs is called domination number ' $\boldsymbol{\gamma}^{\prime}$. A subset $D$ of $\mathrm{V}(\mathrm{G})$ is said to be a Perfect Dominating set if each vertex in $' V-D^{\prime}$ ' is dominated by exactly one vertex of $D$. Among all the Perfect Dominating Sets the one with minimum number of vertices is called Perfect Domination Number which is denoted by $\gamma_{p f}(G)$. 


\section{K.S.P. Sowndarya and Y. Lakshmi Naidu}

The chessboard domination problems are said to be the origin of study of dominating sets in graphs [8]. The Queens Domination problem was considered as one of the most interesting and difficult among the chessboard problems. Jaenisch [5] considered these domination problems for the first time in 1862. Rouse Ball [2] in 1892 gave domination and independent domination of queens for $n \leq 8$. Ahrens [1] in 1910 extended this by providing $\boldsymbol{\gamma}\left(\boldsymbol{Q}_{\boldsymbol{n}}\right)$ for $n \leq 13$ and $n=17, \boldsymbol{i}\left(\boldsymbol{Q}_{\boldsymbol{n}}\right)$ for $n \leq 12$. Thus the experimental values for $n \leq 17$ have been modified by Cockayne [4] for values of $n=5,6,7$. Welch (private communication to Cockayne [4]) was the first to derive upperbound for Queens Domination, and after great attention over this area for years Spencer (private communication to Cockayne [4]) had shown that $\gamma\left(\boldsymbol{Q}_{\boldsymbol{n}}\right) \geq \frac{\boldsymbol{n}-\mathbf{1}}{\mathbf{2}}$ which was then later improved by Weakley[11] by finding the bounds for value of $\mathrm{n}$ of the form $n=4 k+1$ by showing that $\boldsymbol{\gamma}\left(\boldsymbol{Q}_{\mathbf{4 k + 1}}\right) \geq \mathbf{2} \boldsymbol{k}+\mathbf{1}$ for all $k \geq 0$ which was then extended by Burger, Cockayne and Mynhardt [3] giving $\gamma\left(Q_{n}\right) \leq \frac{31 n}{54}+O(1)$. The first upperbound for $i\left(Q_{n}\right)$ was given by Cockayne [4] with $i\left(Q_{n}\right) \leq 0.705 n+0.895$ which was then improved by Grinstead, Hahne, and Vanstone [7] to $i\left(Q_{n}\right) \leq \frac{2 n}{3}+O(1)$. Fricke et al. [6] made a conjecture where $\gamma\left(Q_{n}\right)=i\left(Q_{n}\right)$ for sufficiently large values of $n$. The lower (upper) domination number $\gamma(G)(\Gamma(G))$, independence number $i(G)(\beta(G))$, and irredundance number $\operatorname{ir}(G)(\operatorname{IR}(G))$ are respectively minimum (maximum) cardinalities among minimal dominating, maximal independent and maximal irredundant sets of $\mathrm{G}$. These six parameters were explained in detail in the literature [9] and they satisfy the inequality

$$
i r(G) \leq \gamma(G) \leq i(G) \leq \beta(G) \leq \Gamma(G) \leq I R(G)
$$

In [12] Yaglom and Yaglom showed that $\gamma\left(B_{n}\right)=n$ and $\beta\left(B_{n}\right)=2 n-2$. The values for the Rooks and Kings was also shown in [12] by Yaglom and Yaglom as

$$
\gamma\left(R_{n}\right)=\beta\left(R_{n}\right)=n ; \quad \gamma\left(K_{n}\right)=\left\lfloor\frac{n+2}{3}\right\rfloor^{2}
$$

$\beta\left(K_{n}\right)=\left(\frac{n}{2}\right)^{2}$ when $n$ is even, $\left(\frac{n+1}{2}\right)^{2}$ when $n$ is odd. In this paper we will find the values of perfect domination number for Bishops, Kings and Rooks graphs on a $n \times n$ chessboard. In section 3 we show the placement of minimum number of Bishops that can be placed on the board so that each cell is dominated exactly once and cover the entire chessboard which does not exceed ' $2 n-1$ '. Similarly, in section 4 and 5 , we show the placement of minimum number of Kings and Rooks to be placed in the chessboard satisfying the condition of perfect domination respectively.

\section{Defining placement of cells}

For any positive integer $n$, a chessboard of order $n$ has $n$ columns, each consisting of $n$ square cells which are considered to run from bottom to top as mentioned in [10], where the cell in the lower left-hand corner is numbered $\langle 1,1\rangle$ and the other cells are numbered as $\langle i, j\rangle$. Here $i$ denote a row and $\mathrm{j}$ denotes a column.

Every square in the board of Bishops graph $\boldsymbol{B}_{\boldsymbol{n}}$ is intersected by two lines through the cell which are diagonals; whereas, in Rooks graph $\boldsymbol{R}_{\boldsymbol{n}}$ is intersected by two lines, one 
Perfect Domination for Bishops, Kings and Rooks Graphs On Square Chessboard

being the column and the other being the row. A column is said to be Empty if there is no chess piece placed in that particular column. Kings Graph $\boldsymbol{K}_{\boldsymbol{n}}$ is intersected by four lines which are at a distance of one from the King placed, where two being the diagonals and one being the row and the other being the column.

\section{Perfect domination number for bishops graph $\gamma_{p f}\left(B_{n}\right)$}

Theorem 1. For $n \geq 4$, perfect domination number $\gamma_{p f}\left(B_{n}\right)=2 n-1$.

Proof: We first define the placement of Bishops on the chessboard $\boldsymbol{B}_{n}$ for $n$. Place the ' $2 n-1$ ' Bishops in the following order. First we place ' $n$ ' Bishops in each cell of the main diagonal i.e., $\langle i, j\rangle$ where $i=j$ starting from the cell $\langle 1,1\rangle$ and moving from bottom to top. Next place ' $n-1$ ' Bishops in the diagonal below or above the main diagonal i.e., $\langle i, i+1>$ where $i=1, \ldots, n-1$, or $\langle i, i-1>$ where $i=2, \ldots \ldots, n$.

This placement will cover the entire chessboard with each cell being dominated exactly once and gives minimum number of Bishops required to satisfy the condition of perfect domination number $\boldsymbol{\gamma}_{\boldsymbol{p} f}\left(\boldsymbol{B}_{\boldsymbol{n}}\right)$. If we place the Bishops in a single row or a column then there will be a chance of a cell being dominated by more than one Bishop. So, to avoid the cell being dominated more than once we first place the Bishops diagonally in the main diagonal. Also, note that all the cells in the diagonal must be placed with bishops because leaving at least one cell in the diagonal will give us a cell which will be dominated by more than one Bishop. For $n=3$ the value of $\gamma_{p f}\left(B_{3}\right)=3$ and the placement of Bishops for $B_{3}$ is given below in fig. 1 .

The following fig. 2 shows the placement of Bishops for $n=6$ where $\gamma_{p f}\left(B_{6}\right)=11$.

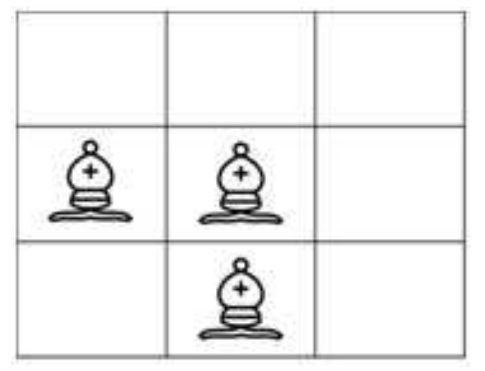

Figure 1: Perfect domination for $\mathrm{B}_{3}$

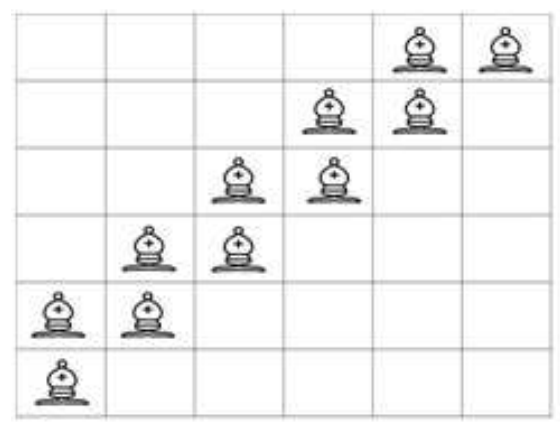

Figure 2: Perfect domination for $\mathrm{B}_{6}$ 
K.S.P. Sowndarya and Y. Lakshmi Naidu

\section{Perfect domination number for kings graph $\gamma_{p f}\left(K_{n}\right)$}

Theorem 2. For $n \in N, \gamma_{p f}\left(K_{n}\right)=\left[\frac{n+2}{3}\right\rfloor^{2}$.

Proof: We first define the placement of Kings on the chessboard $\boldsymbol{K}_{\boldsymbol{n}}$ for different values of ' $n$ ', where $n \geq 3$

We have three different cases for the placement of Kings. If $n=3 k$ i.e., if $\mathrm{n}$ is divisible by 3 as in [12], then divide the board into $(k)^{2}$ boxes of width 3 and height 3 , and place one King in each box in such a way that it covers the entire board and no cell gets dominated more than once.

If $n=3 k+1$, i.e., if $n$ leaves remainder 1 when divided by 3 , then divide the board into $(k+1)^{2}$ boxes as follows: First, divide the board with height 2 from top and bottom and divide the boxes remaining in between with height 3 . Now, divide the board at width of 2 from $1^{\text {st }}$ column and also from the last column, and divide the remaining in between columns at a width 3 such that we will get $(k+1)^{2}$ boxes. Next, we place one King in each box so that it satisfies the condition of perfect domination.

If $n=3 k+2$, i.e., if $n$ leaves remainder 2 when divided by 3 then divide the board into $(k+1)^{2}$ boxes as follows: First, partition the board at height 2 from the top and remaining boxes at height 3 . then divide the board again column wise with first partitioning the board at width 2 from last column and the remaining columns at width 3 such that we get $(k+1)^{2}$ boxes and then place one King in each of the boxes so that it satisfies perfect domination.

As we know that each king on $n \times n$ chessboard is said to attack its own square and its neighboring squares, and also by definition of perfect domination, note that the distance between one king to another must be maintained by at least 2. Fig. 3, 4 and 5 show the placement of perfect domination of Kings graph $\boldsymbol{K}_{n}$ for $n=6,7$, and 8 where each cell is dominated exactly by one King. Combining these different values of $n$ gives the exact value of $\gamma_{p f}\left(K_{n}\right)=\left\lfloor\frac{n+2}{3}\right\rfloor^{2}=\gamma\left(K_{n}\right)$ as mentioned in [12]. Thus we say that $\gamma\left(K_{n}\right)=\gamma_{p f}\left(K_{n}\right)$.

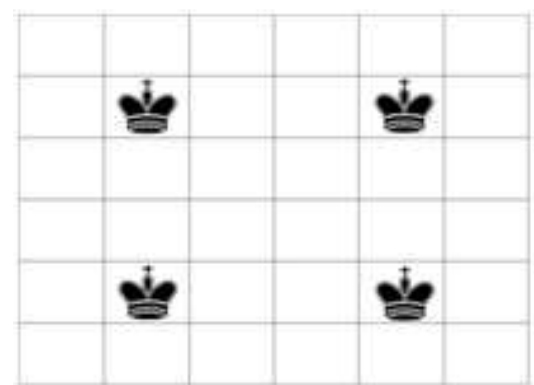

Figure 3: Perfect domination for $\mathrm{K}_{6}$ 
Perfect Domination for Bishops, Kings and Rooks Graphs On Square Chessboard

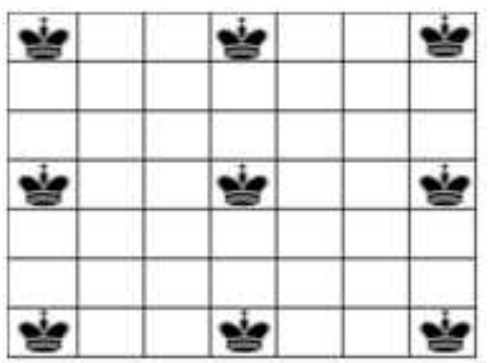

Figure 4: Perfect domination for $\mathrm{K}_{7}$

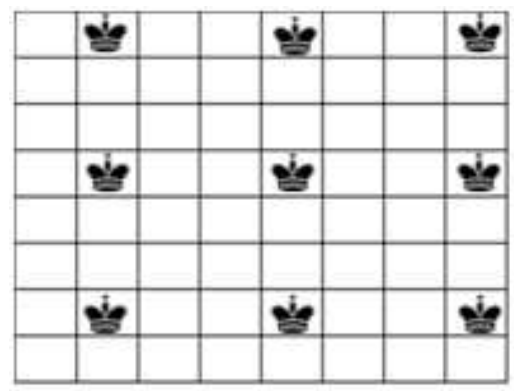

Figure 5: Perfect domination for $\mathrm{K}_{8}$

\section{Perfect domination number for rooks graph $\gamma_{\boldsymbol{p} \boldsymbol{f}}\left(\boldsymbol{R}_{\boldsymbol{n}}\right)$}

Theorem 3. $\forall n, \gamma_{p f}\left(R_{n}\right)=\gamma\left(R_{n}\right)=n$.

Proof: The placement of Rooks for perfect domination is same as the placement for domination number as in [12] i.e., as the movement of the Rooks is limited to rows and columns ' $n$ ' Rooks should be placed on $n \times n$ chessboard. Therefore, we place all the ' $n$ ' Rooks either in a single row or a column, but not in any other position like diagonal as done for domination, because placing in any other positions would give us at least one cell which would be dominated more than once contradicting the concept of perfect domination. Thus the perfect domination number in Rooks graph $\boldsymbol{R}_{n}$ is ' $n$ ' for all $n$. The following fig. 6 shows the placement for $n=5$.

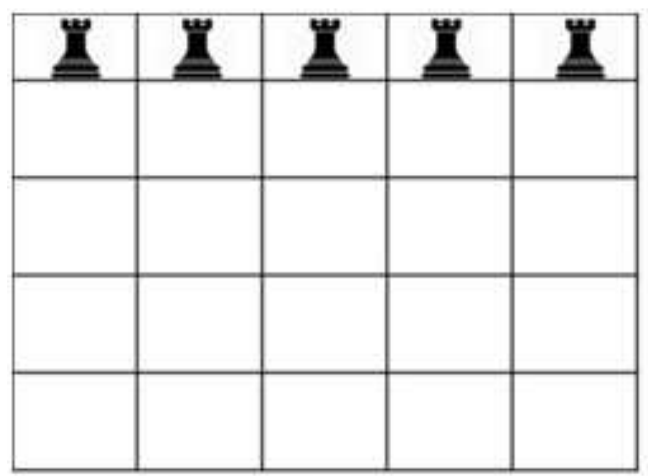

Figure 6: Perfect domination for $\mathrm{R}_{5}$ 


\section{K.S.P. Sowndarya and Y. Lakshmi Naidu}

\section{Conclusion}

In this paper the exact values and placement for the Bishops, Kings, and Rooks on the square chessboard using the perfect domination parameters were presented. We would like to extend this work on various other chessboards.

Acknowledgement. We pay our sincere thanks to all the authors, professors, and experts for their contributions, and also would like to thank the reviwers for their useful suggestions.

\section{REFERENCES}

1. W.Ahrens, Mathematische Unterhaltungen und Spiele, Liepzig-Berlin, 1910.

2. W.W.R.Ball, Mathematical Recreations and Problems of Past and Present Times, MacMillan, London, 1892.

3. A.P.Burger, E.J.Cockayne and C.M.Mynhardt, Domination and irredundance in the Queens' graph, Discrete Mathematics, 163 (1997) 47-66.

4. E.J.Cockayne, Chessboard domination problems, Discrete Math., 86 (1990) 13-20.

5. C.F.de Jaenisch, Applications de l'Analyse Mathematique au Jeu des Echecs, Petrograd, 1862.

6. G.H.Fricke, S.M.Hedetniemi, S.T.Hedetniemi, A.A.Macrae,C.Wallis, M.S.Jacobson, H.W.Martin and W.D.Weakley, Combinatorial problems on chessboards: a brief survey, Proc. $7^{\text {th }}$ Internat. Conf. on Graph Theory, Combinatorics, Algorithms and Applications, Kalamazoo, Michigan, 1992.

7. C.M.Grinstead, B.Hahne and D.Van Stone, On the Queen domination problem, Discrete Math., 86 (1991) 21-26.

8. T.W.Haynes, S.T.Hedetniemi and P.J.Slater, Fundamentals of Domination in Graphs, Marcel Dekker, New York, 1998.

9. S.T.Hedetniemi and R.C.Laskar, A bibliography on dominating sets in graphs and some basic definitions of domination parameters, Discrete Math., 86 (1990) 257-277.

10. W.F.D.Theron and G.Geldenhuys, Domination by queens on a square beehive, Discrete Math., 178 (1998) 213-220.

11. W.D.Weakley, Domination in the queen's graph, in: Y.Alavi, A.J.Schwenk (Eds.), Graph Theory, Combinatorics, and Algorithms, Vol.2, Wiley-Interscience, New York, 1995, pp.1223-1232.

12. A.M.Yaglom and I.M.Yaglom, Challenging Mathematical Problems with Elementary Solutions, Holden-Day, Inc., San Francisco, 1964. 